muss man einen neuen Beweisgrund dafür finden, dass Prof. Seh äfer erst nach der Publication meiner Arbeit deutliche Vorstellnngen über die Rolle der Leucocyten bei der Resorption des Fettes erhalten und sich. unzweifelhaft von der Theilnahme dieser Zellen an dem Processe überzeugt hat. Anders kann man sich nicht erklären, warum Herr Prof. Schäfer im Jahre 1885 in einem internationalen Journale ein Factum zur allgemeinen Kenntniss bringt, welches er schon im Jahre 1876 gefunden hat und für welches er damals nicht für nöthig hielt, dass es den engen Rahmen seines Lehrbuches iberschreite. In diesem Umstande kann man von Seiten des Herrn Prof. Schäfer das Zugeständniss finden, dass er gegenwärtig selbst die Ungeeignetheit des damals im Jahre 1876 eingeschlagenen Publicationsweges einsiebt, d. h. dasselbe, was auch ich ihm zu beweisen suchte. Statt meinen Namen ironisirend mit dem Processe der Fettresorption in Verbindung zu bringen, müsste mir eigentlich Herr Prof. Schäfer dankbar sein, dass ich ihm die Gelegenheit geboten habe auch seinen Namen an den Process der Fettresorption anschmiegen zu können.

\title{
Thierisches Dextran, ein neuer gummiartiger Stoff in den Excrementen einer Blattlaus.
}

$$
\text { Von }
$$

\section{Leo Liebermann.}

Vor einiger Zeit übergab mir Herr Dr. G. Horváth, Vorstand der hiesigen Phylloxera-Versuchsstation, behufs Untersuchung Excremente einer Blattlaus und theilte mir zur Orientirnng folgendes mit:

"Schizoneura lanuginosa Hart., gallenbewohnende Blattlaus, auf Ulmen in ganz Europa verbreitet und nicht eben selten."

"Die Blattlaus erzengt im Frühjahr durch ihren Stich und fortwährendes Saugen an den Enden der Seitenzweige der Ulmenbäume grosse, birnförmige, wallnussgrosse und öfters noch grössere Gallen, in welchen dann das Mutterthier sammt ihrer zahlreichen Nachkommenschaft lebt. Die Galle ist anfangs geschlossen und die Excremente der Blattlans, welche in Form von kleinen, klaren Tröpfehen aus ihrem After hervorsickern, bleiben in der Galle." 
„Da die Blattlänse aus ihrem Körper zugleich einen wolligen Flaum ausschwitzen und damit ganz umgeben sind, so werden auch diese Tröpfehen von dem wolligen Secret umschlossen und rollen umber wie Quecksilbertropfen. Später fliessen die Tröpfchen immer mehr zusammen und werden grösser."

„Wenn die Galle im Sommer an mebreren Punkten aufreisst, damit die schon geflügelten Thiere ausfliegen köunen, fällt ein Theil der Excrement-Klümpehen auf die Erde. Ein Theil bleibt aber in den Gallen und erstarrt dort. So findet man sie gegen Herbst in den verlassenen und ganz vertrockneten Gallen."

Das mir von Herrn Dr. Horváth freundlichst zur Verfügung gestellte Material stammt von Farkasd im Comitate Pest, aus dem Versuchsweingarten der kgl. Phylloxera-Versuchsstation. Es bildet zum Theil schmutzigbraune oder schwarzbraune unregelmässige, stellenweise wie verschimmelt aussehende Klumpen, zum Theil mehr weniger runde oder ovale, linsen- bis erbsengrosse Körner, von harzigem Aussehen und gelbbrauner, brauner oder schwarzbrauner Farbe. Manche glänzen wie Pech.

lch habe gefunden, dass diese Klumpen und Körner der Hauptmasse nach aus einer Art von stark rechtsdrehendem Gu m m i bestehen. Darstellung und Eigenschaften sollen in Folgendem beschrieben werden.

Die Klumpen und Körner wurden, so gut es ging, im Mörser zerkleinert und dann mit dest. Wasser gekocht.

Es entstand eine dunkel grünlichbraune, trübe Lösung, welche durch Thierkohle filtrirt ziemlich lichtgelb wurde. Sie war jedoch noch immer truibe and konnte durch Filtration nicht gereinigt werden, wohl aber durch längeres Schütteln mit gebrannter Mag. nesia und nachheriges Filtriren.

Die nun völlig klare, gelbe Lösung mit Salzsäure bis zur stark sauren Reaction und dann mit viel 96\% igem Alkohol versetzt, gab eine dichte, weisse Trübung. Nach mehrtägigem Stehen im bedeckten Becherglase klärte sich die Flüssigkeit fast vollständig. Am Boden des Glases fand sich eine Schicht eines weissen, durchscheinenden Körpers, ähnlich einer dünnen Schicht weissen Wachses.

Die Flüssigkeit wurde von dieser nun einfach abgegossen, einige Male mit Alkohol nachgewaschen und der Körper in Form 
einer dünnen, biegsamen Platte, von der Grösse des Bodens des Becherglases, mit dem Glasstabe heransgehoben.

Ueber Schwefelsäure getrocknet, zerbröckelte die im feuchten Zustande gummiartig klebrige Masse und liess sich leicht pulvern. - Ibre Reactionen sollen weiter unten angegeben werden.

Die Analyse der über Schwefelsäure bis zu Gewichtsconstanz getrockneten Substanz ergab folgendes: die Substanz ist stickstofffrei; $0.214 \mathrm{gr}$ Substanz mit $0,002 \mathrm{gr}$ Asche $=0,93 \%$ gaben auf aschefreie Substanz berechnet:

$$
\begin{aligned}
& \mathrm{C}=45,37 \% \\
& \mathrm{H}=6,98,
\end{aligned}
$$

Die feingepulverte Substanz wurde behufs weiterer Reinigung 4 Mal mit Alkohol, hierauf sehr oft mit Aether ausgezogen, in heissem Wasser gelöst und mit Alkohol gefällt.

So gereinigt enthielt sie nur mehr unwägbare Spuren von Asche.

0,1635 gr Substanz gaben:

$$
\begin{aligned}
& \mathrm{C}=45,03 \% \\
& \mathrm{H}=7,33 \%
\end{aligned}
$$

Das Mittel aus diesen 2 Analysen gab demnach folgende procentische Zusammensetzung:

$$
\begin{aligned}
& \mathrm{C}=45,20 \% \\
& \mathrm{H}=7,15, \\
& \mathrm{O}=47,65,
\end{aligned}
$$

worans sich keine gut stimmende Formel berechnen lässt. Am nächsten kommt noch $\mathrm{C}_{6} \mathrm{H}_{10} \mathrm{O}_{5}$, welches $44,44 \% \mathrm{C}$ and $6,17 \% \mathrm{H}$ verlangt.

Die Substanz ist zweifellos ein Kohlehydrat, vielleicht aber verunreinigt mit geringen Mengen eines anderen Körpers.

Man wird da die fractionirte Fällung anwenden miissen, sobald grössere Mengen Materials zur Verfugung stehen.

Zur Bestimmung der specifischen Drehung wurden $0,776 \mathrm{gr}$ der oben erwähnten aschefreien Substanz in $40 \mathrm{~cm}$ Wasser gelöst nod im $200 \mathrm{~mm}-\mathrm{Rohr}$ bei etwa $20^{\circ} \mathrm{C}$. mit dem grossen Apparat von Wild bei Natronlicht die Drehung bestimmt, ohne Rücksicht auf die Dichte der Flïssigkeit, welche ja in diesem Falle keinen nennenswerthen Fehler verursachen konnte. Das Mittel mehrerer Ablesungen betrug $+6,08^{\circ} \cdot[\alpha]_{\mathrm{D}}=+156,7$.

Die weiteren Eigenschaften der Substanz charakterisiren sie 
vollständig als Gummi. Sie klebt wie Gummi, schmilzt zu einer glasigen, beim Erkalten, besonders in dünnen Schichten spröden Masse, löst sich schwer in kaltem, viel leichter in kochendem Wasser zu einer neutralen Flüssigkeit. In Alkohol und Aether ist sie unlöslich.

Beim Verbrennen entwickelt sie einen Geruch wie brennendes Papier. Die wässrige Lösung mit Kalilauge und Kupfervitriol versetzt und gekocht zeigt keine Spur von Reduction. Auch Magisterium Bismuthi wird nicht reducirt.

Kali und Kupfervitriollösung erzeugen einen etwas grïnlichblauen gallertig-klumpigen Niederschlag, welcher abfiltrirt und mit Salzsäure zersetzt eine Lösung gibt, welche bei Zusatz von viel Alkohol wieder weisses Gummi fallen lässt.

Bleizuckerlösung erzeugt in der wässrigen Lösung des Körpers keinen Niederschlag. Versetzt man ausser mit Bleizucker auch noch mit Schwefelwasserstoff, so fällt kein Schwefelblei aus, sondern es entsteht eine braune Lösung, welche mit derselben Farbe filtrirt.

Versetzt man eine wässrige Lösung vorerst mit so viel Alkohol, dass noch kein bleibender Niederschlag entsteht, so erzeugt Bleizucker einen Niederschlag, der sich aber in Wasser wieder löst.

Bleiessig gibt einen Niederschlag.

Jodlösung färbt nicht roth, auch gibt die Substanz keine Reaction mit Pikrinsäure und Kali.

Mit Natronlauge gekocht wird die Lösung erst nach längerer Zeit lichtgelb.

Kocht man längere Zeit mit verdünnter Schwefelsäure unter fortwährendem Ersatz des verdampfenden Wassers, so bekommt man eine Flüssigkeit, welche die Trommer'sche Reaction gibt.

Stumpft man die Säure fast vollständig ab, versetzt mit etwas Presshefe und lässt $1 \mathrm{Tag}$ bei Zimmertemperatur stehen, so verliert die Flüssigkeit die Fähigkeit, Kupferoxyd zu reduciren.

Die Substanz ist daher eine Gummiart, welche wahrscheinlich einen gährungsfähigen Zucker liefert.

Ihre Zusammensetzung unterscheidet sie von der Arabinsäure $\left(\mathrm{C}_{12} \mathrm{H}_{22} \mathrm{O}_{11}\right)$, ihr sehr starkes Rotationsvermögen von allen bekannten Gummiarten.

Ich schlage vor, diesen Körper "thieriscbes Dextran“ zu nennen und zwar darum, weil die Bezeichnung „thierisches 
Gummi“, welche näher läge, bekanntlich schon durch einen K̈örper occupirt ist, welchen Landwehr ${ }^{1}$ ) als Bestandtheil des Macins beschrieben hat. Das Wort Dextran riuhrt von Scheibler ${ }^{2}$ ) her, der damit eine sehr stark rechtsdrehende Gummiart $\left.\left(\alpha=+223^{0}\right)^{3}\right)$ bezeichnet, welche er in unreifen Rüben entdeckt hat.

Sieht man vom ,thierischen Gummi" Landwehr's ab, von welehem es ïbrigens noch zweifelhaft ist, ob es wirklich Gummi ist, da dessen Lösung die charakteristische Kupferreaction (mit schwefelsaurem Kupferoxyd und iiberschuissigem Kali entsteht in einer Gummilösung ein etwas gallertiger, klumpiger Niederschlag) ${ }^{4}$ ) nicht gibt, sondern wie Landwehr angibt, eine hellblaue Lösung, so ist es nur Staedeler, der in einer Fussnote zu seiner Arbeit über Fibrin, Spongin, Chitin ${ }^{5}$ ) im Jahre 1859 mittheilt, er habe in Maikäfern, Seidenraupen und in den Kiemen des Flusskrebses ein arabinartiges Gummi gefunden, ohne jedoch auf die nähere Beschreibung desselben einzugehen.

Ich glaube daher, dass der von mir besehriebene Fall der erste ist, bei welchem wirkliches Gummi als thierische Substanz unzweifelhaft nachgewiesen erscheint. Kürzlich bat Landwehr wieder eine $\Lambda$ rbeit über thierisches Gummi publicirt (dieses Archiv, Bd. 40, p. 21) in welcher gesagt wird, man habe sich daran zu erinnern, dass das thierische Gummi sich gegen Fällungsmittel ganz wie Pflanzengummi verhält (p. 36). Ich habe schon bemerkt, dass dies aus Landwehr's früheren Publicationen nicht so ganz unbedingt hervorgeht und weiss nun nicht, ob Landwehr's neuester Ausspruch sich auch auf neuere Untersuchungen stïtzt. Jedenfalls standen diese bezïglich der Kupferreaction in Widerspruch mit den fribheren, vorausgesetzt, dass sich die auf p. 37 beschriebene Reaction auf thierisches Gummi bezieht. Es ist mir nämlich nicht ganz klar geworden, ob der Autor den Leser nur an die bekannten Pflanzengummi-Reactionen erinnern will oder aber behauptet, dass sie auch sämmtlich bei thierischem Gummi mit positivem Erfolg angestellt wurden.

Eine Zusammenstellung verschiedener Gummianalysen findet

1) Zeitschr. f. physiol. Chemie. 8. 122.

2) Wagner's Jahresber. f. chem. Technologie, 1875, 790.

3) 1. c. p. 792 .

4) Barfoed, Lehrb. der org. qual. Analyse, 1881, p. 230.

5) Li ebig's Annalen CXI, p. 26. 
man in Gmelin-Kraut's Handbuch der Chemie, 4. Aufl.; Org. Chemie, IV. Bd., I. Abth., p. 641.

Bei der Ausfïhrung dieser Arbeit bin ich von Hrn. E. Rei chenhaller unterstuitzt worden.

\title{
Die Bewegungsempfindung.
}

\author{
Zweite Mittheilung. \\ Von \\ Hermann Anbert.
}

Hierzu Tafel VII.

Bei meinen Untersuchungen über die Bewegungsempfindung ${ }^{1)}$ hatte ich einen grossen Unterschied für die Winkelgeschwindigkeiten gefunden, welche erforderlich sind, um eine Bewegungsempfindung auszulösen, je nachdem sich ruhende Objecte im Gesichtsfelde neben dem bewegten Objecte befinden, oder ruhende Objecte möglichst ausgeschlossen sind und die Aufmerksamkeit auf die bewegten Objecte concentrirt wird.

Ein vollständiger Ausschluss der ruhenden Objecte war dabei aber nicht bewerkstelligt worden und ich glaubte, einen solchen dadurch erreichen zu können, dass ich sichtbare, sich bewegende Objecte im uibrigens absolut finsteren Raume beobachtete. Mit der 'Anordnung darauf gerichteter Versuche beschäftigt wurde ich aber veranlasst, zuvor ein Moment zu untersuchen, welehes von wesentlicher Bedeutung für das Zustandekommen der Bewegungsempfindung ist, nämlich den Einfluss der Augenbewegungen beim Beobachten bewegter Objecte. Durch eine briefliche Mittheilung: des Herrn Professor v. Fleisehl (Fle is chl von Marxow) in Wien wurde ich in sehr liebenswürdiger Weise auf einen Aufsatz desselben, welcher mir unbekannt geblieben war, aufmerksam gemacht.

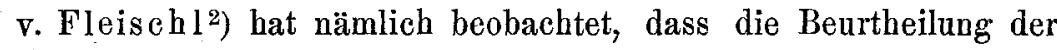

1) Dieses Archiv, Bd. XXXIX, 1886, S. 347.

2) E. v. Fleischl, physiologisch-optische Notizen (2. Mittheilung), Sitzungsberichte der Wiener Akademie, Bd. 86, III. Abth. 1882, Heft 1, S. 17, V. 